\title{
Sun Tracking Method for Fractal Concentrator Panels
}

\author{
Ilia Katardjiev \\ Department of Electrical Engineering, Uppsala University \\ ilia.katardjiev@angstrom.uu.se
}

30 January 2021

\begin{abstract}
The transmittance of Fractal Concentrators (FC) exhibits a slow and steady decline for incidence angles beyond the acceptance angle. This feature can be employed to track the Sun by the FC solar panel itself without the need of additional tracking devices which is the subject of this work. Of specific interest are FC solar panels at relatively high geometrical concentrations $C_{g}$ and, hence, with relatively small acceptance angles.
\end{abstract}

\section{Introduction}

FC concentrators have been recently proposed [1,2] for concentrating sunlight. Such concentrators consist of an array of refractive optical elements (OE) of conical shape and separated by small gaps of a lower refractive index material. Consequently, rays entering an $O E$ initially propagate through total internal reflection until they eventually lose forward component and start bifurcating through concurrent refraction/reflection events at the interfaces creating in the process a cascade of fractal rays. Thus most generally, such concentrators operate under the principle "one ray in - more than one ray out" which results in a spatially homogeneous exit flux[3] amongst other advantages. At low geometrical concentrations FC concentrators are shown to have a sufficiently large acceptance angle eliminating thus the need for sun tracking. This is not the case at high concentrations. Thus, the acceptance angle $\theta_{a}$ of the concentrator is given by:

$\theta_{a}=\operatorname{asin}\left[\frac{n_{1}}{n_{2 \sqrt{C_{g}}}}\right]-\varepsilon=\theta_{\text {th }}-\varepsilon$

where $n_{1}$ is the refractive index of the OEs, $n_{2}$ is the refractive index of the gaps, $\varepsilon$ is a function of the angular aperture of the concentrator and the acceptance angles of the OEs, while $\theta_{\text {th }}$ represents the theoretical limit. Strictly speaking, the angular acceptance aperture of the concentrator represents the common intersection between the angular acceptance apertures of the individual OEs. In practice, $\theta_{\mathrm{a}}$ is readily calculated numerically for any specific geometry. Further, it has been also shown[3] that the transmittance of an FC concentrator at $100 \mathrm{X}$ declines slowly and steadily for incidence angles $\theta$ in the interval:

$\theta_{\mathrm{a}}<\theta<\theta_{\text {th }}$

as illustrated in Figure 1. 


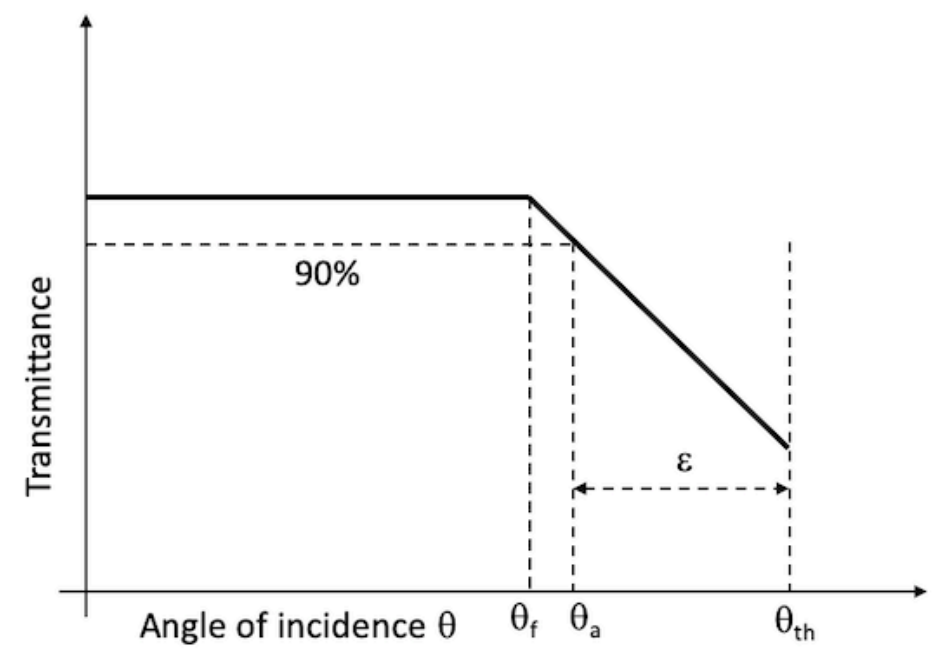

Figure 1. Typical behaviour of the transmittance.

Specifically, it shows a typical behaviour of the FC transmittance as a function of the incidence angle $\theta$. Notably, the transmittance is flat for incidence angles smaller than $\theta_{f}$ beyond which it declines steadily.

\section{Method for sun tracking}

FC concentrators are typically to be used in a flat panel configuration as schematically illustrated in Figure 2.

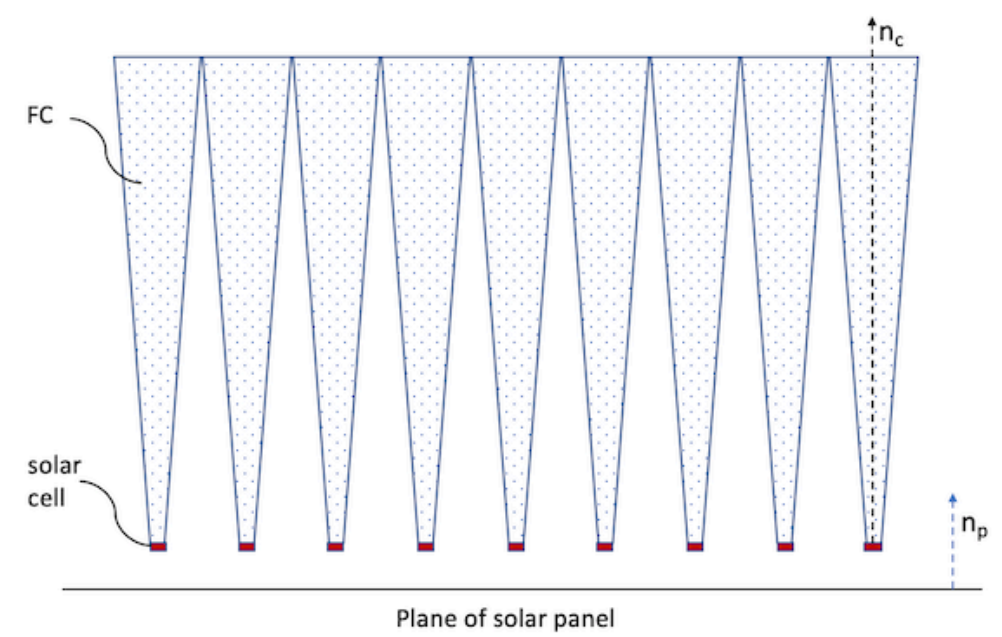

Figure 2. Flat panel configuration of FC concentrators.

Specifically, the FC concentrators are arranged tightly in a planar array with their axes $\mathrm{n}_{\mathrm{c}}$ parallel to the normal $n_{p}$ to the panel plane. Solar cells are optically connected to the exit apertures of each concentrator. As noted above each FC concentrator consists in turn of an array of OEs but in view of eq. (1) we limit the number of OEs to six in order to maximize the acceptance angle (minimize $\varepsilon$ ). Assuming also that the OEs represent truncated triangular pyramids results in a hexagonal periphery of the FC concentrators. Figure 3 represents a top view of a solar panel with such FC concentrators. 


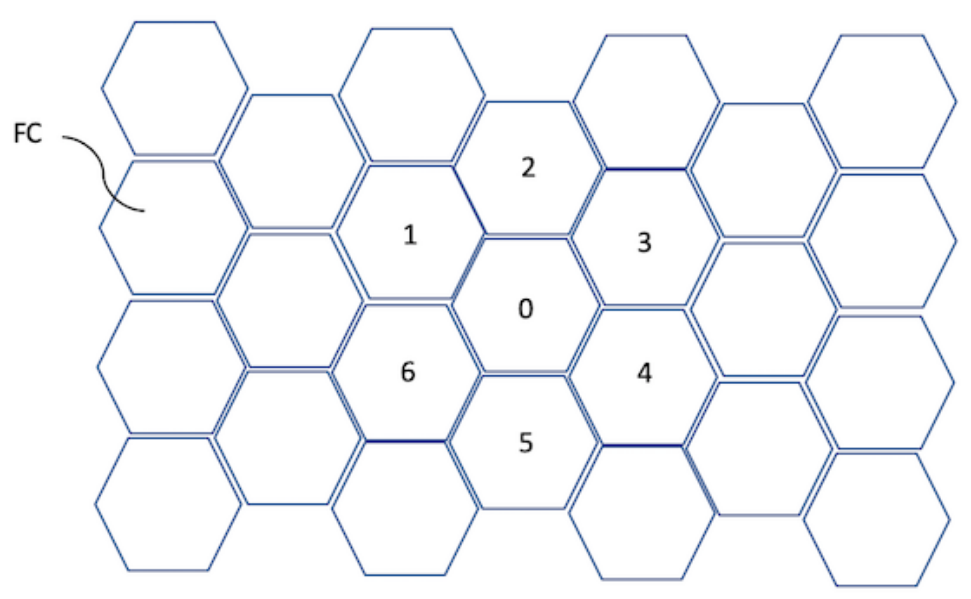

Figure 3. Top view of an FC solar panel.

We now tilt the axes $n_{c}$ of concentrators 1 trough to 6 in Fig. 3 by bringing their exit apertures closer to the central FC concentrator denoted as " 0 " such that the axis $\mathrm{n}_{\mathrm{c}}$ of any of the six concentrators lies in a plane containing the normal $n_{p}$. Finally, the tilt angle is chosen to be $\theta_{\mathrm{f}}$ while the azimuthal separation between two neighbouring concentrators naturally is $60^{\circ}$. Figure 4 illustrates the definition of the tilt angle where only two of the six concentrators are illustrated for clarity.

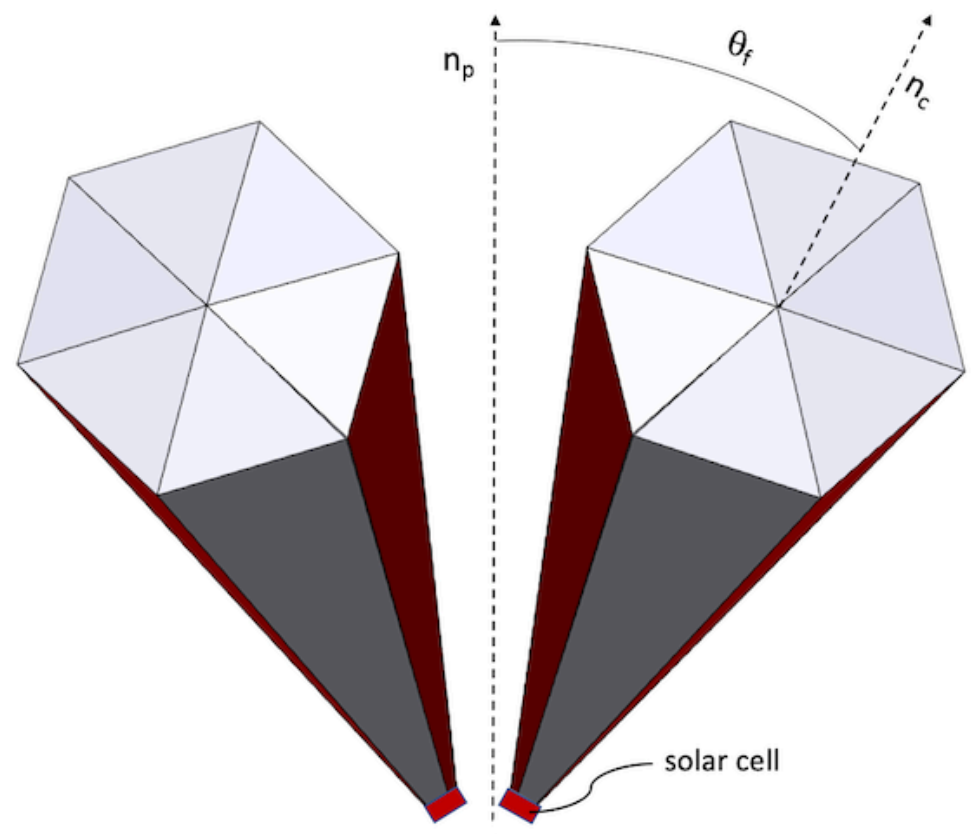

Figure 4. Illustration of the tilt angle $\theta_{\mathrm{f}}$.

It is clear from the above construction that the FC concentrators 1 through to 6 will be operated at the edge of the flat region of the transmittance at perfect alignment. Hence, any misalignment will produce a decreasing voltage signal by the solar cells whose FC concentrators are misaligned by an angle exceeding $\theta_{\mathrm{f}}$. Hence, from knowledge of the positions of the six concentrators in the plane of the solar panel one can readily calculate the gradient vector of the output voltages (voltage gradient) in the same plane and which vector 
together with the normal $n_{p}$ determine the plane of rotation. The actual angle of rotation is obtained from a standard calibration procedure. Hence, the angular positioning of the solar panel is achieved by first positioning it from prior knowledge of geo-position and time of the year followed by fine tuning using the above method. In this way the panel stays aligned at all times without needing additional tracking devices. As a matter of fact, the panel in this way stays locked onto the Sun more or less at the centre of the angular acceptance aperture of the panel itself since any disbalance (voltage gradient) greater than a predefined margin is immediately detected and subsequently compensated. Note that compensation is always possible as long as the misalignment of at least two of the 6 concentrators is smaller than $\theta_{\text {th. }}$

The concentrators 1 through to 6 were intentionally chosen to be clustered together in order to minimize the chance of false readings produced by partial shadowing from clouds and the like. Several such clusters may be distributed over the panel surface to further minimize this probability. Any loss of efficiency due to the misalignment of tilted FC concentrators is negligible since the areal aperture of the individual FC concentrators is much smaller (by orders of magnitude) than the total area of the panel.

\section{Conclusions}

The inherent behaviour of the transmittance of FC concentrators has been employed to devise a method for indirect tracking of the Sun by monitoring the output voltages at selected solar panel concentrators tilted appropriately for maximum response. The overall performance of the solar panel is not affected.

\section{References}

[1] I. Katardjiev, A fractal concentrator of direct and indirect light, Clean Energy, 2020, https://academic.oup.com/ce/article/4/4/305/5983284

[2] I. Katardjiev, Optical characterization of a fractal concentrator, https://doi.org/10.33063/diva-430393, 2021

[3] I. Katardjiev, Evaluation of a Fractal Concentrator of Light at 100X, submitted to CPV-17, http://cpv-17.org, 2021

\section{Note:}

This is an Open Access article distributed under the terms of the Creative Commons Attribution Non-Commercial License (http://creativecommons.org/licenses/by-nc/4.0/) 